\title{
Role of inhibitor of growth 4 in the suppression of human melanoma cells through the Fas/FasL-mediated apoptosis pathway
}

\author{
LIMIN CAI ${ }^{1 *}$, HAIYAN LI ${ }^{2 *}$, CUI CHEN $^{1}$, XUE CHENG $^{1}, \mathrm{YU} \mathrm{WANG}^{1}, \mathrm{JING} \mathrm{LIU}^{1}$, \\ YONGCHEN WANG ${ }^{1}$ and LIJUN HAO ${ }^{3}$
}

\begin{abstract}
${ }^{1}$ Department of Dermatology, The First Affiliated Hospital of Harbin Medical University; ${ }^{2}$ Department of Dermatology,
The Affiliated Tumor Hospital of Harbin Medical University; ${ }^{3}$ Department of Plastic Surgery, The First Affiliated Hospital of Harbin Medical University, Harbin, Heilongjiang 150010, P.R. China
\end{abstract}

Received November 11, 2015; Accepted October 27, 2017

DOI: $10.3892 /$ ijmm.2017.3274

\begin{abstract}
Melanoma, the most aggressive form of skin cancer, is notoriously resistant to all current available therapies. Inhibitor of growth 4 (ING4), a novel member of the ING family of proteins, has previously been shown to play a critical role in the development of multiple tumors by regulating apoptosis, proliferation, cell cycle progress, migration and invasion. However, the functional role of ING4 in human melanoma remains unclear. To fully understand its potential role in human melanoma, in the present study, lentivirus (LV)-ING4 and LV-ING4-short hairpin RNA were constructed and transfected into human melanoma A375 cells. First, the effect of overexpressing or downregulating ING4 on the apoptosis of the transfected melanoma cells and cluster of differentiation (CD) $3^{+} \mathrm{T}$ cells was investigated. In the present study, we found that the late apoptotic cells, and not the early apoptotic cells, were more in LV-ING4 group compared with LV-control, and both the early and late apoptosis of $\mathrm{CD}^{+} \mathrm{T}$ cells was significantly observed in A 375 cells transfected with LV-ING4 compared with LV-control. Importantly, it was determined whether the overexpression of ING4 significantly induce apoptotic cell death via Fas/FasL (Fas death receptor/FasL) pathway activation and downregulation of poly(ADP-ribose) polymerase, caspase- 3 and caspase- 8 in the melanoma cells
\end{abstract}

Correspondence to: Dr Yongchen Wang, Department of Dermatology, The First Affiliated Hospital of Harbin Medical University, 21 Youzheng Street, Nangang, Harbin, Heilongjiang 150010, P.R. China E-mail: yongchenwang123@163.com

Dr Lijun Hao, Department of Plastic Surgery, The First Affiliated Hospital of Harbin Medical University, 21 Youzheng Street, Nangang, Harbin, Heilongjiang 150010, P.R. China

E-mail: 1ijunhao019@163.com

*Contributed equally

Key words: melanoma, inhibitor of growth 4, Fas/FasL, apoptosis and $\mathrm{CD}^{+} \mathrm{T}$ cells. These results demonstrated that overexpression of ING4 can induce the apoptosis of melanoma cells and $\mathrm{CD}^{+} \mathrm{T}$ cells through signaling pathways such as the Fas/FasL pathway, and that ING4 gene therapy for melanoma treatment is a novel approach.

\section{Introduction}

Melanoma is one of the most lethal forms of skin tumors, responsible for $>80 \%$ of all diagnosed skin cancer-related mortalities (1). Melanoma is a serious public health problem in all countries throughout the world due to its unfavorable prognosis and the limited treatments available (2). Despite advances in melanoma treatment, with several novel targeted therapies, as a result of the special characteristics and usual resistance to standard chemotherapy, there is no systemic and effective therapy that has a clear effect on the overall survival of patients with malignant melanoma (3). Although our understanding of the molecular biology of malignant melanoma has increased in recent years, the molecular mechanism of melanomagenesis is not completely understood.

Melanoma cells are highly resistant to traditional chemotherapeutic and radiotherapeutic treatments (4). Immunotherapies (involving cancer vaccines, adoptive immunotherapy, antibodies and cytokines) focus on alternative therapies (5). The challenge is to translate successful results from immune modulation trials into clinical meaningful results in phase 2 or randomized phase 3 drug trials (6). Combining cancer vaccines with the adoptive transfer of $\mathrm{T}$ cells has been shown to increase the levels of circulating tumor antigen-specific regulatory $\mathrm{T}$ cells in patients, however, these approaches produce only a few patient's clinical responses (7). These observations in melanoma support the fact that tumor resistance to immune system-mediated destruction may be the main process in the tumor microenvironment (7). To improve the concepts of immune-based treatments, a better understanding of the local and systemic tumor-resistance mechanisms is important in melanoma patients.

The five members of the inhibitor of growth (ING) gene family are ING1, ING2, ING3, ING4 and ING5. The ING 
family have garnered attention due to their putative roles as tumor suppressor genes $(8,9)$. ING4 is a member of the ING family that has been demonstrated to play important roles in numerous cancer-related cellular processes including DNA damage, hypoxia, cell proliferation, apoptosis, the cell cycle, migration and angiogenesis (8). ING4 is located in chromosome 12 p13 and encodes a 249 -amino acid protein containing a conserved $\mathrm{C}$-terminal plant homeodomain finger motif and two nuclear localization signals (10). ING4 expression is reduced in primary melanomas and metastatic melanomas (11). Upregulation of ING4 has been shown to decrease the cell population in $\mathrm{S}$ phase, diminish the colony forming efficiency and induce apoptosis in a p53-dependent manner $(10,11)$. However, the precise role of ING4 in melanoma angiogenesis is unclear.

The Fas death receptor/Fas ligand (Fas/FasL) system is a key regulator of apoptosis (12). The Fas/FasL system, one of the major extrinsic apoptosis signaling pathways, is a key regulator of T-cell apoptosis (13). The Fas/FasL-induced apoptotic pathway is active in early- and intermediate-phase melanomas, but is often impaired in highly metastatic melanomas (14). The lack expression of Fas is correlated with the poor prognosis of malignant melanomas (15). Recent findings revealed that the Fas expression was downregulated and FasL expression was upregulated during melanoma progression (16).

The present study sought to investigate the impact of ING4 on malignant melanoma cells. Firstly, lentivirus (LV)-ING4-short hairpin (shRNA) or LV-ING4 were transfected into human melanoma A375 cells. Next, the effects of ING4 overexpressed or knockdown were determined on the apoptosis of A375 cells and $\mathrm{CD}^{+} \mathrm{T}$ cells. The study aimed to reveal the potential effects of ING4 in the regulation of the apoptosis pathway in A375 cells and CD3 ${ }^{+} \mathrm{T}$ cells. The study also aimed to demonstrate that ING4 can serve as a target for gene therapy in human melanoma cells.

\section{Materials and methods}

Cell and culture. The human melanoma A375 cell line, obtained from the American Type Culture Collection (ATCC, Manassas, VA, USA), was cultured in RPMI-1640 medium (Gibco; Thermo Fisher Scientific, Inc., Waltham, MA, USA), supplemented with $10 \%$ fetal bovine serum (MinHai Bio-Engineering, Co., Ltd., Lanzhou, China) and 1\% penicillin-streptomycin (Invitrogen; Thermo Fisher Scientific, Inc.) in a humidified atmosphere at $37^{\circ} \mathrm{C}$, in the presence of $5 \% \mathrm{CO}_{2}$ and $95 \%$ air. After selection of infected A375 cells, the CD $3^{+}$ T cells were co-cultured with A375 cells in WT, LV-control, LV-ING4-shRNA and LV-ING4 groups separately.

Construction of overexpression or knockdown ING4 vectors in A375 cells. pcDNA3.1-ING4 was constructed as described previously (17). Lentiviral vector (pLKO.1) constructs containing ING4 cDNA or shRNA sequences for ING4 and an empty vector were purchased from Open Biosystems; GE Healthcare Dharmacon, Inc. (Lafayette, CO, USA). Lentiviral constructs were used to transiently transfect 293FT packaging cells along with $3 \mu \mathrm{g}$ VSV-G pseudoviral particles. At $16 \mathrm{~h}$ post-transduction, the supernatant was replaced by Opti-MEM medium supplemented with HEPES (Invitrogen; Thermo
Fisher Scientific, Inc.). Viral supernatant was harvested from the 293FT cells at $48 \mathrm{~h}$ post-transfection and filtered to remove non-adherent cells. Subconfluent A375 cells were infected by centrifugation at $300 \mathrm{x}$ g for $5 \mathrm{~min}$ at $4^{\circ} \mathrm{C}$ using virus-containing medium and $8 \mu \mathrm{g} / \mathrm{ml}$ polybrene. Infected A 375 cells were selected for using $2 \mu \mathrm{g} / \mathrm{ml}$ puromycin starting at $24 \mathrm{~h}$ after the initial infection. The cells were identified at the protein levels using western blot analysis.

Preparation of $\mathrm{CD}^{+} \mathrm{T}$ cells. Peripheral blood mononuclear cells (PBMCs) were isolated from the peripheral blood of healthy donors using Ficoll density gradient centrifugation at $300 \mathrm{x} \mathrm{g}$ for $10 \mathrm{~min}$ at $20^{\circ} \mathrm{C}$. Activation and expansion of anti-CD3 T cells from PBMCs at 14 days post-centrifugation was performed as previously described (18). CD3 ${ }^{+} \mathrm{T}$-cell expansion resulted in a product increase of $98.85 \pm 1.06 \%$ on day $15 . \mathrm{CD}^{+} \mathrm{T}$ cells were cultured in RPMI-1640 containing $10 \%$ fetal calf serum (Invitrogen; Thermo Fisher Scientific, Inc.). The research protocol was approved by the Biomedical Research Ethics Committee of the First Affiliated Hospital of Harbin Medical University (2014-R-034).

Analysis of apoptosis with Annexin V/7-aminoactinomycin D (7-AAD) staining. Annexin V-fluorescein isothiocyanate (FITC) and 7-AAD (BD Biosciences, San Jose, CA, USA) were used for detection of apoptotic cells in the melanoma A375 cells and $\mathrm{CD}^{+} \mathrm{T}$ cells, according to the manufacturer's protocols, and then analyzed by a dual-staining protocol with fluorescence-activated cell sorting using FACScan (BD Biosciences, Franklin Lakes, NJ, USA). Cell populations $\left(1 \times 10^{6}\right)$ were labeled with Annexin $\mathrm{V}$ and 7 -AAD (BD Biosciences) following the provider's procedure, and analyzed by flow cytometry.

In vivo model. A total of $20 \mathrm{BALB} / \mathrm{c}$ nude mice were purchased from the Shanghai Laboratory Animal Center (Shanghai, China). Mice were allowed free access to sterilized water and food, and were housed in individual ventilated cages at $23 \pm 5^{\circ} \mathrm{C}$ under a 12-h light/dark cycle. The A375 cells were resuspended in serum-free DMEM at a concentration of $1 \times 10^{7}$ cells $/ \mathrm{ml}$. A total volume of $0.2 \mathrm{ml}$ cell suspension (total $2 \times 10^{6}$ cells) was then injected subcutaneously into the right anterior armpit of nude mice to establish a xenograft model. The 20 injected mice were randomly divided into 4 groups: WT, LV-control, LV-ING4-shRNA and LV-ING4 (n=5 per group). The experimental protocol was approved by the Animal Care and Use Committee of the First Affiliated Hospital of Harbin Medical University.

Western blot analysis. Melanoma A375 cells or $\mathrm{CD}^{+} \mathrm{T}$ cells were lysed in buffer containing $150 \mathrm{mmol} / 1 \mathrm{NaCl}, 1 \% \mathrm{NP}-40$, $50 \mathrm{mmol} / 1$ Tris (pH 8.0) and 20\% glycerol, and normalized to total protein concentration using Bio-Rad protein assay reagent (Bio-Rad Laboratories, Inc., Hercules, CA, USA). Whole cell proteins $(30 \mu \mathrm{g})$ were separated by $10 \%$ sodium dodecyl sulfate-polyacrylamide gel electrophoresis (SDS-PAGE) and transferred to PVDF membranes using the Bio-Rad electrotransfer system (Bio-Rad Laboratories, Inc.). The filters were hybridized with ING4 (sc-135742), poly(ADP-ribose) polymerase (PARP; sc-136208), caspase-8 (sc-6136), caspase-3 
(sc-271759), Fas (sc-4856) and FasL (sc-71096) (all 1:1,000 dilution; Santa Cruz Biotechnology, Inc., Dallas, TX, USA) for $1 \mathrm{~h}$ at room temperature. Anti-glyceraldehyde 3-phosphate dehydrogenase (GAPDH; 1:1,000 dilution; sc-47724; Santa Cruz Biotechnology, Inc.) was used as a loading control. The membrane was blocked by $5 \%$ fat-free milk for $1 \mathrm{~h}$ at room temperature and then incubated with the appropriate primary antibody diluted in $3 \%$ BSA solution at $4^{\circ} \mathrm{C}$ overnight. After incubation with DyLight dye-conjugated secondary antibodies (cat. no. 35518; 1:10,000; Thermo Fisher Scientific, Inc.) for $1 \mathrm{~h}$ at room temperature, blots were scanned by the Odyssey Western Detection system (LI-COR Biosciences, Lincoln, NE USA), followed by quantification with ImageStudio software (LI-COR Biosciences).

Immunohistochemistry. Immunohistochemistry (IHC) was performed as described previously (19). Fine sections (4-5 $\mu \mathrm{m})$ were prepared from formalin-fixed tissue sections on poly-L-lysine coated glass slides, and then stained with anti-PARP, -caspase- 8 , -caspase-3, -Fas and -FasL by IHC. Sections were evaluated and scored independently by an experienced pathologist unaware of the treatment group. At least 5 fields per slide were randomly chosen for analysis of IHC. The IHC was evaluated according to the intensity of reactivity using a 4-tier system: 0 , no staining (-); 1 , weak staining (+); 2 , moderate staining $(++)$; and 3 , strong staining $(+++)$.

$R N A$ extraction and RT-qPCR. Total RNA was extracted by using the TRIzol reagent (Life Technologies, Carlsbad, CA, USA) according to the manufacturer's instructions. RNA was reverse transcribed into cDNA using the PrimeScript II 1st strand cDNA Synthesis kit (Takara, Shiga, Japan). qPCR was performed onanABIStep-Oneplus machine using SYBR-Green qPCR Master Mix (Biotool, Jupiter, FL, USA). Primers used are as follows: ING4 forward, ATGACAGCTCTTCCAGCAA and reverse, AGAAACTGTGTTGGAATCCAAG; GAPDH forward, AATCCCATCACCATCTTCCA and reverse, TGGACTCCACGACGTACTCA. GAPDH was used as endogenous control and $2^{-\Delta \Delta C t}$ method was used to calculate the fold change.

Statistical analysis. All experiments were repeated at least three times, and data are expressed as the mean \pm standard error of the mean. Comparisons among multiple groups were determined by one-way analysis of variance followed by Tukey's post-hoc test. Prism6 (GraphPad Software, Inc., La Jolla, CA, USA) was use to perform statistical analysis. $\mathrm{P}<0.05$ was considered to indicate a statistically significant difference.

\section{Results}

Expression of ING4 in melanoma A375 cell transfectants. The expression of ING4 is $98 \%$ in dysplastic nevi, and then significantly decreased to 67 or $53 \%$ in primary melanomas and metastatic melanomas (11). After the wild-type, non-targeting lentiviral, ING4 lentiviral small hairpin RNA (LV-ING4-shRNA) and lentiviral pcDNA3.1-ING4 (LV-ING4) were transfected into A375 melanoma cell line, respectively, the expression of ING4 was evaluated by RT-PCR and
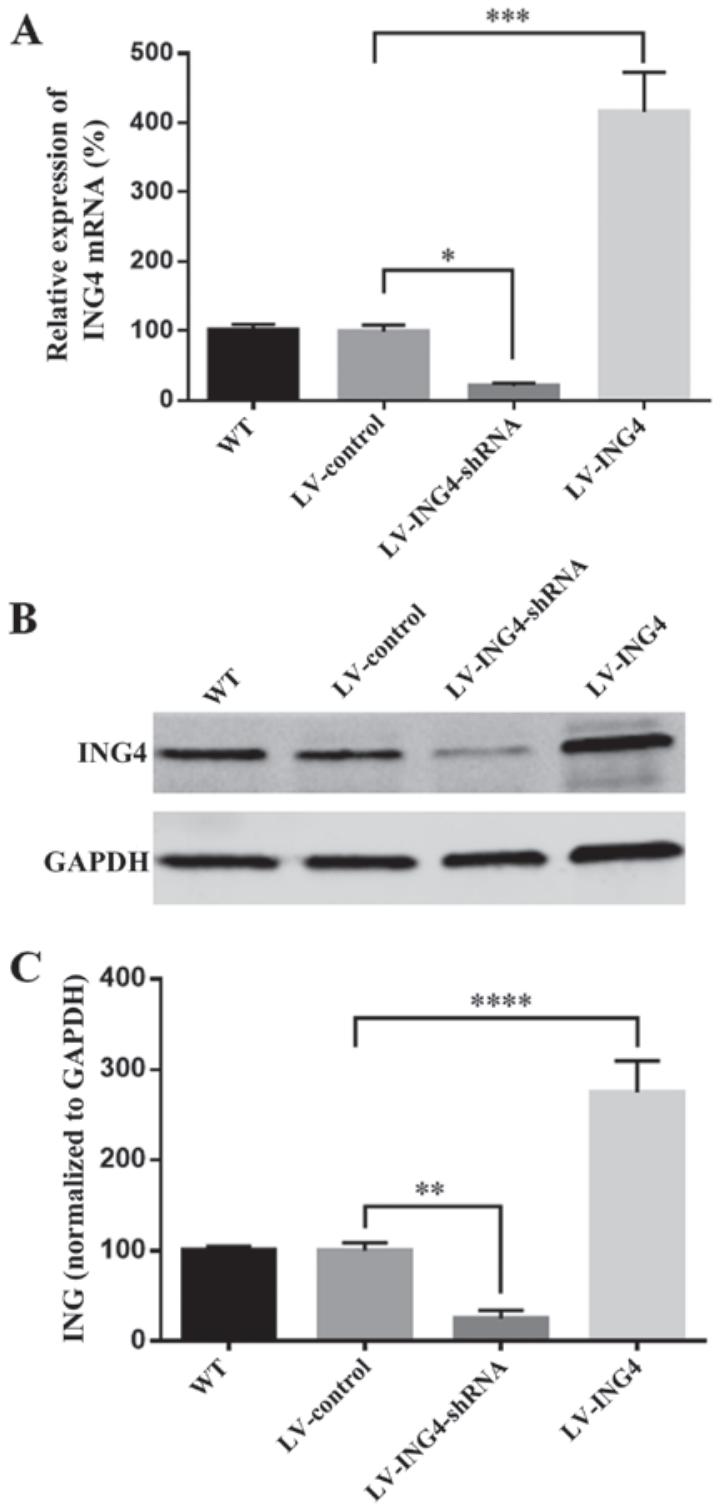

Figure 1. ING4 gene knockdown or overexpression in the melanoma A375 cell line. Cells were transfected with ING4 shRNA or ING4 plasmid, and a negative control was included. The expression of ING4 was measured by (A) reverse transcription-polymerase chain reaction and (B) western blotting. (C) ING4 protein expression in A375 melanoma cells was normalized by GAPDH. ING4, inhibitor of growth 4; GAPDH, glyceraldehyde 3-phosphate dehydrogenase; shRNA, short hairpin RNA; LV, lentivirus; WT, wild-type.

western blot analysis. The overall knockdown efficiency of LV-ING4-shRNA was $80 \%$ (Fig. 1A) at the mRNA level and $70 \%$ at the protein levels (Fig. 1B and C). However, there was a significant increase in ING4 mRNA of $415 \%$ and protein of $275 \%$ in the A375/LV-ING4 group compared with the A375/LV-control (Fig. 1).

Overexpression of ING4 enhances apoptosis of melanoma A375 cells and $\mathrm{CD}^{+} \mathrm{T}$ cells. To examine the effect of ING4 on the A375 and T-cell co-culture system, $\mathrm{CD}^{+} \mathrm{T}$ cells were isolated from the blood samples of healthy donors. Flow cytometry analysis was performed to confirm the purity of $\mathrm{CD}^{+}$ $\mathrm{T}$ cells from the $\mathrm{A} 375$ and $\mathrm{CD}^{+}$T-cell co-culture (Fig. 2).

Next, to determine whether the effect of ING4 in A375 melanoma cells to enhance the apoptosis of $\mathrm{A} 375$ cells or $\mathrm{CD}^{+}$ 

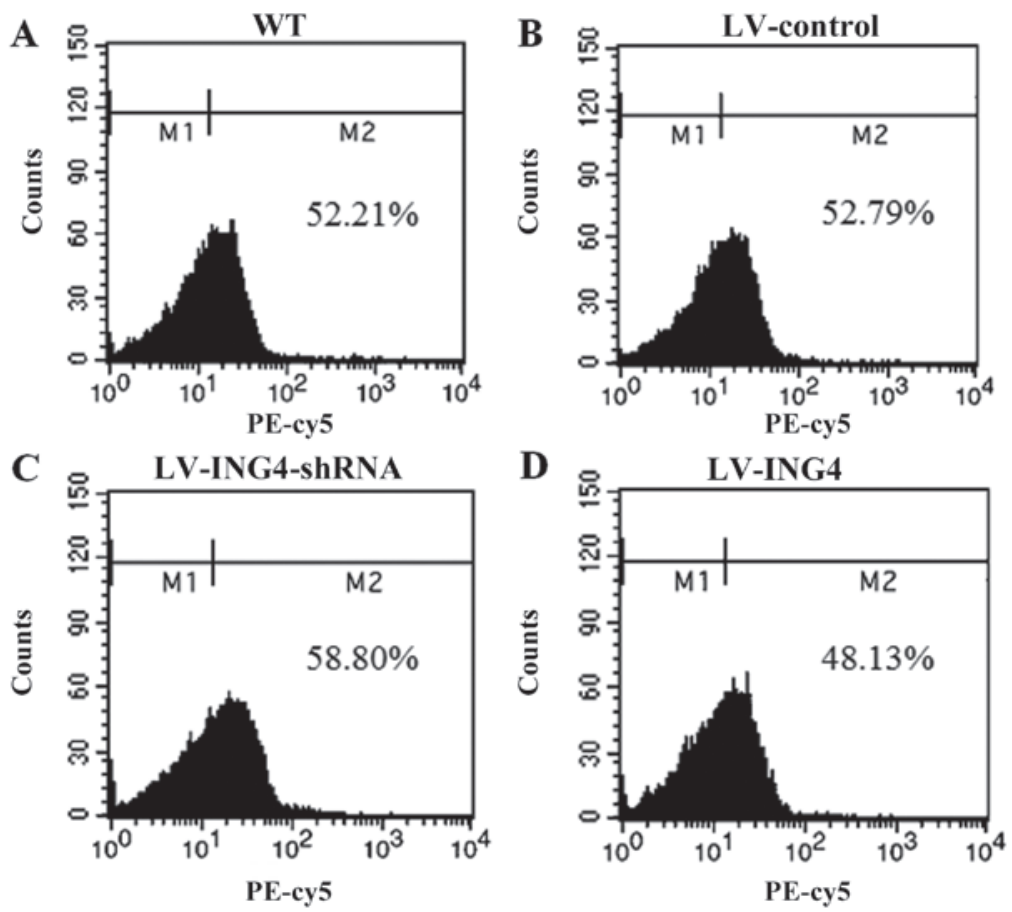

Figure 2. Percentage of $\mathrm{CD}^{+} \mathrm{T}$ cells in the A375 cell and T-cell co-culture was measured by staining with anti-CD3 and fluorescence-activated cell sorting analysis. (A) WT, (B) LV-control, (C) LV-ING4-shRNA and (D) LV-ING4. ING4, inhibitor of growth 4. WT, wild-type; PE, phycoerythrin; LV, lentivirus; ING4, inhibitor of growth 4; shRNA, short hairpin RNA.

T cells, the A375 cells were treated with wild-type, LV-control, LV-ING4-shRNA or LV-ING4, and then co-culture with CD3 ${ }^{+}$ $\mathrm{T}$ cells at $48 \mathrm{~h}$; double staining with Annexin V-FITC/PI was used to detect the cell apoptosis (Fig. 3). The percentage of early apoptotic cells (Annexin V-positive/7-AAD-negative) was $6.24 \pm 0.75 \%$ for the wild-type group, $6.02 \pm 1.52 \%$ for the LV-control group, 7.01 $\pm 1.42 \%$ for the LV-ING4-shRNA group and $4.68 \pm 0.89 \%$ for the LV-ING4 group. In addition, the percentage of late apoptotic cells (Annexin V-positive/7-AAD -positive) was $5.76 \pm 1.37 \%$ for the wild-type group, $8.41 \pm 1.47 \%$ for the LV-control group, $5.98 \pm 1.21 \%$ for the LV-ING4-shRNA group and $54.37 \pm 6.13 \%$ for the LV-ING4 group (Fig. 3A).

Similar results were obtained with $\mathrm{CD}^{+} \mathrm{T}$ cells from the A 375 and $\mathrm{CD}^{+}$T-cell co-culture system. As shown in Fig. 3B, the apoptosis of $\mathrm{CD}^{+} \mathrm{T}$ cells was significantly different in the A375 cells transfected with LV-ING4 (the early apoptotic cells, $16.47 \pm 2.63 \%$; the late apoptotic cells, $43.20 \pm 4.96 \%$ ) compared with that in the cells transfected with the LV-control (the early apoptotic cells, $6.21 \pm 0.87 \%$; the late apoptotic cells, $7.76 \pm 1.49 \%$ ); however, there was no significant difference compared with the LV-ING4-shRNA group in the A375 and $\mathrm{CD}^{+} \mathrm{T}$ cell co-culture system (Fig. 3B). These results suggest that increased ING4 expression in melanoma cells leads to increased lymphocyte apoptosis.

ING4 regulates the protein expression involved in the apoptosis pathway in $\mathrm{CD}^{+}$and melanoma A375 cells. In order to investigate the molecular mechanisms in the apoptosis of $\mathrm{CD}^{+}$and A375 melanoma cells, the effects of ING4 on the expression of target proteins involved in the apoptosis of $\mathrm{CD}^{+}$and melanoma A375 cells was investigated. The protein expression levels of PARP, caspase- 8 and caspase-3, which were involved in cell apoptosis according to western blot analysis, were determined. The protein expression of Fas and FasL was also examined. The results showed that increased ING4 expression reduced the PARP, caspase- 8 and caspase-3 levels, but significantly increased the Fas and FasL protein expression in the $\mathrm{CD}^{+} \mathrm{T}$ cells and the melanoma A375 cells (Fig. 4). The levels of PARP, caspase-8, caspase-3, Fas and FasL were also analyzed in nude mice livers by IHC. Higher expression of Fas and FasL, as well as lower expression of PARP, caspase- 8 and caspase- 3 was observed in the livers of the LV-ING4 group compared with other groups (Fig. 5).

\section{Discussion}

ING4, a novel member of the conserved ING family, has been identified as a critical tumor suppressor in various cancer types. Previous studies suggested that knockdown of ING4 gene expression or ING4 gene deletion was associated with the progression or poor prognosis of high-grade tumors, such as those in lung cancer $(20,21)$, brain tumors $(19,22,23)$ and those in ovarian cancer (24). In addition, previous findings have shown that ING4 expression is significantly reduced in human malignant melanomas, and that through regulating different signaling pathways, ING4 level is closely associated with the proliferation, apoptosis, invasion, metastasis and survival of malignant melanomas cells (11). Meanwhile, a previous study showed that ING4 could significantly reduce tumor cell growth via the regulation of cell cycle progression according to experiments where exogenous ING4 was transfected into a lung cancer cell line (17). We hypothesized that ING4 could play an inhibitory role by inducing cell apoptosis in human malignant melanomas. The present study was designed to elucidate the possible mechanisms of the ING4 expression involved in the induction of A375 cell apoptosis. 

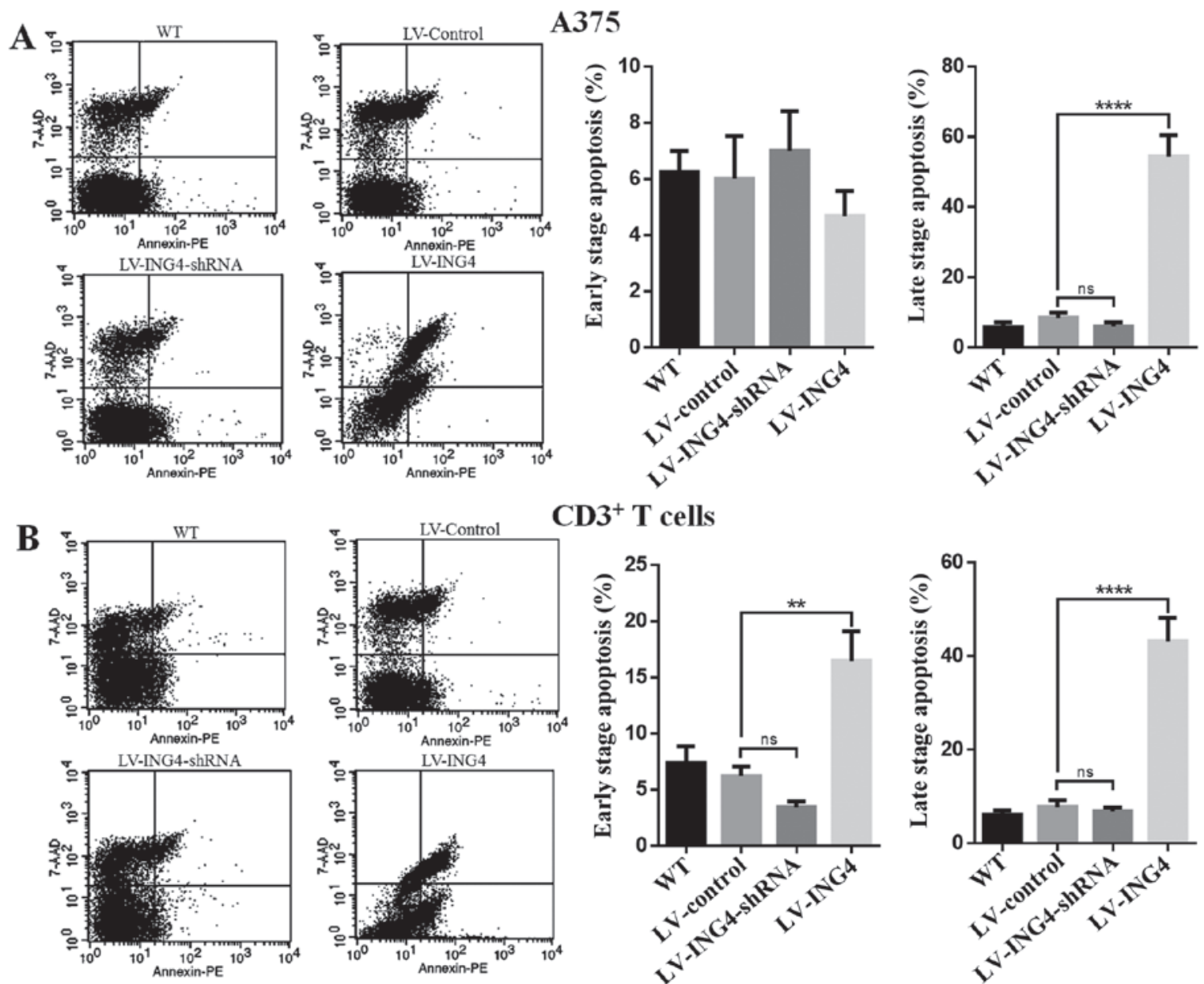

$\mathrm{CD3}^{+} \mathrm{T}$ cells
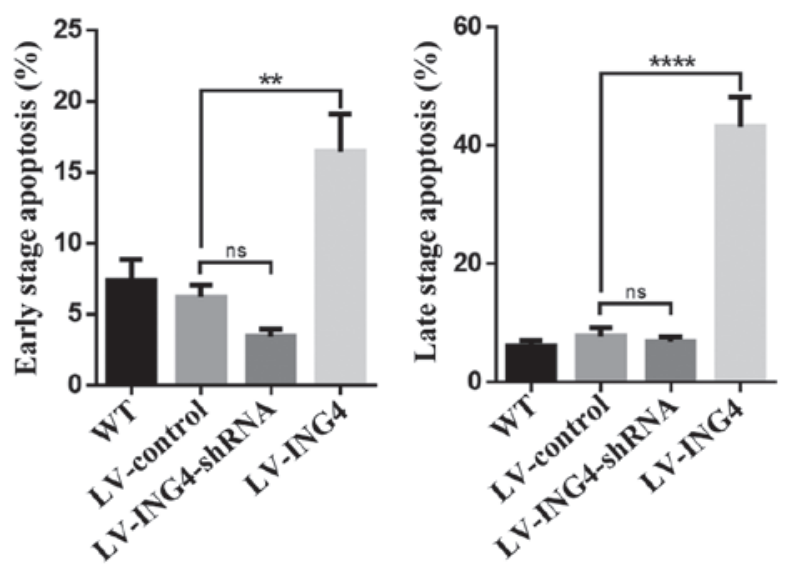

Figure 3. Overexpression of ING4 enhances apoptosis of melanoma A375 cell lines and CD3 ${ }^{+} \mathrm{T}$ cells. The (A) melanoma A375 cells and (B) $\mathrm{CD} 3^{+} \mathrm{T}$ cells were stained with Annexin V-FITC and 7-AAD following transfection with WT, LV-control, LV-ING4-shRNA or LV-ING4. Fluorescence-activated cell sorting analysis of the cells was performed at $48 \mathrm{~h}$ post-transfection with WT, LV-control, LV-ING4-shRNA or LV-ING4. Percentages represent Annexin V-positive/7-AAD-negative (early apoptotic) and Annexin V-positive/7-AAD-positive cells (late apoptotic). Experiments were repeated three times. ${ }^{* *} \mathrm{P}<0.01$ and ${ }^{* * * *} \mathrm{P}<0.0001$ vs LV-control. ING4, inhibitor of growth 4; FITC, fluorescein isothiocyanate; 7-AAD, 7-aminoactinomycin D; WT, wild-type; shRNA, short hairpin RNA; CD, cluster of differentiation; PE, phycoerythrin; LV, lentivirus; ns, not significant.

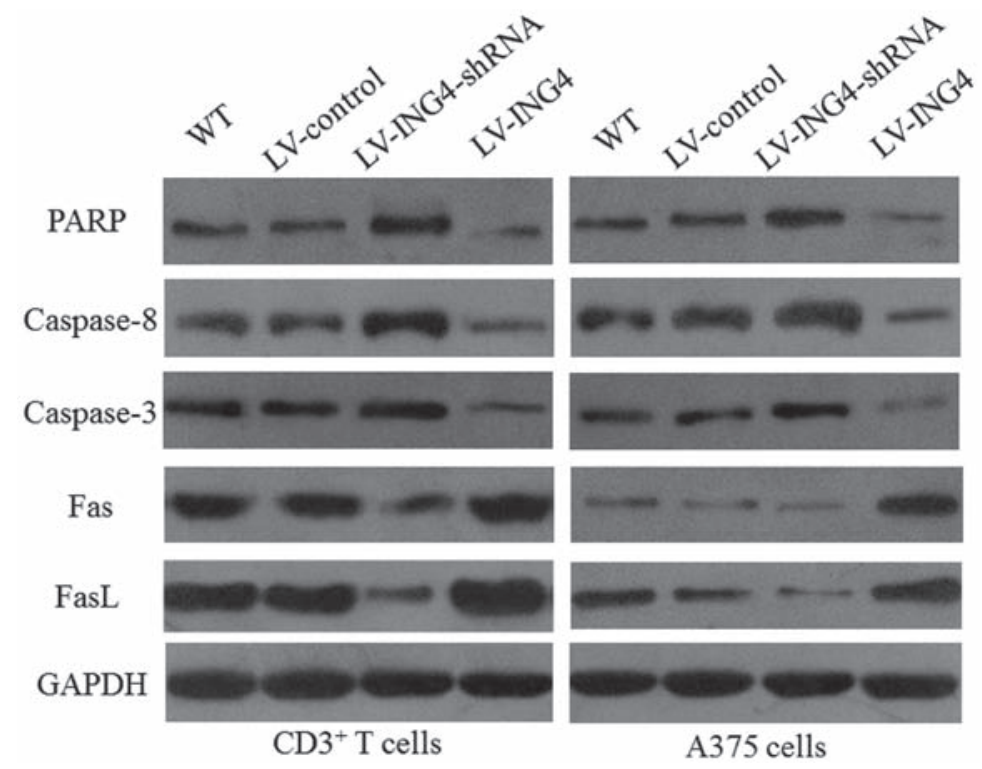

Figure 4. ING4 regulates the expression of apoptotic proteins. Total proteins were extracted from differently treated melanoma A375 cell lines and assessed by western blotting. Upregulation of ING4 decreased PARP, caspase-8 and caspase-3 expression, and increased the expression of Fas and FasL. Expression of GAPDH was used as an internal control. ING4, inhibitor of growth 4; PARP, poly(ADP-ribose) polymerase; LV, lentivirus; shRNA, short hairpin RNA; WT, wild-type; Fas, Fas death receptor; FasL, Fas ligand; GAPFH, glyceraldehyde 3-phosphate dehydrogenase; CD, cluster of differentiation. 


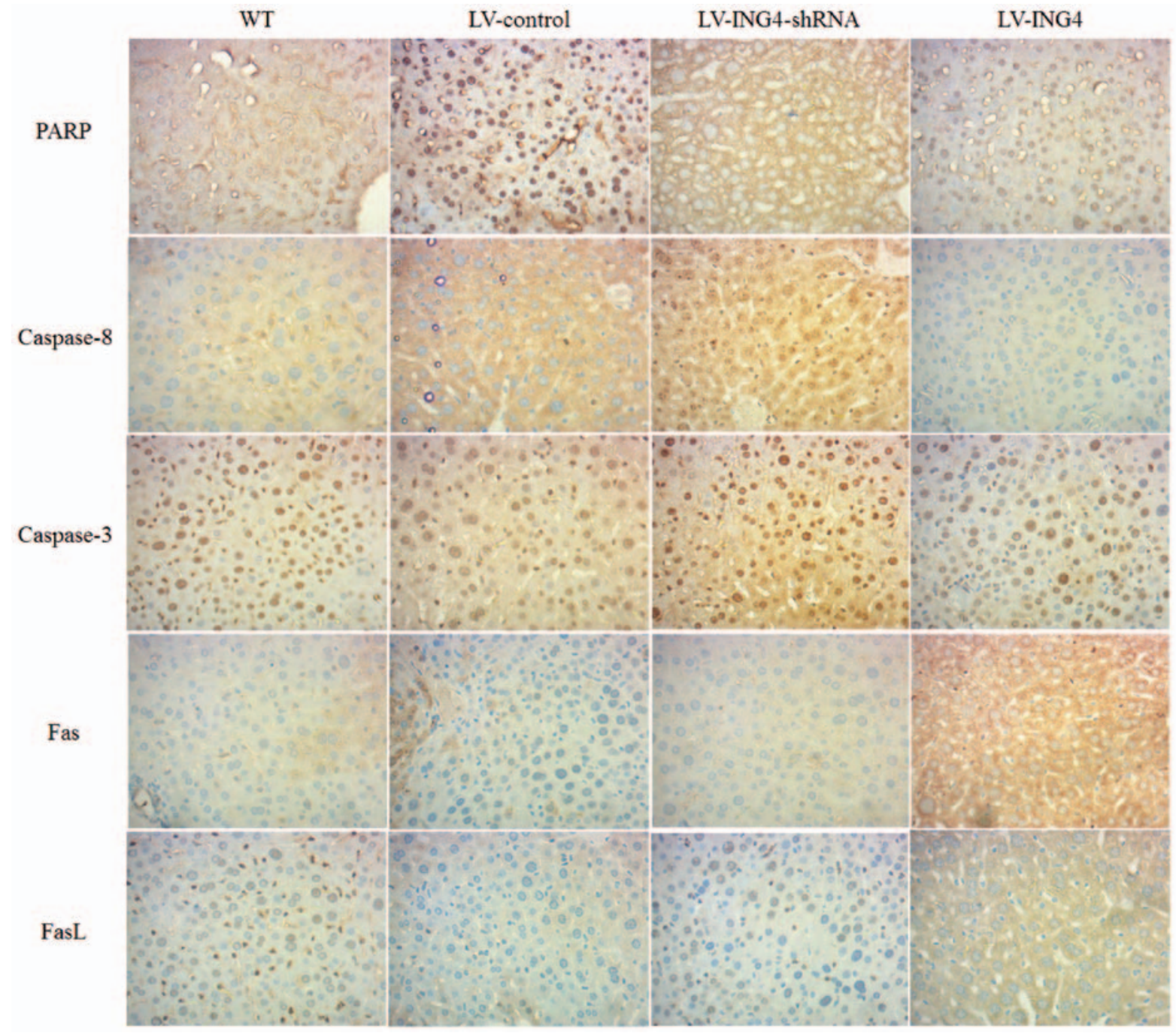

Figure 5. Expression of PARP, caspase-8, caspase-3, Fas and FasL in nude mouse livers assessed by immunohistochemistry in the WT, LV-control, LV-ING4-shRNA and LV-ING4 groups. ING4, inhibitor of growth 4; PARP, poly(ADP-ribose) polymerase; LV, lentivirus; shRNA, short hairpin RNA; WT, wild-type; Fas, Fas death receptor; FasL, Fas ligand.

Apoptosis or programmed cell death consists of the ordered disassembly of the cell from within, as opposed to necrosis or accidental cell death (25). Cellular immunity plays an important role in the antitumor immune response. $\mathrm{CD}^{+}$ $\mathrm{T}$ cells recognize peptides of exogenous antigens, which are presented by major histocompatibility complex class II molecules. Through the release of cytokines, $\mathrm{CD}^{+} \mathrm{T}$ cells activate natural killer cells, enhance the cytotoxicity of effector cells and increasing the sensitivity of cytotoxic $\mathrm{T}$ lymphocytes to target cells, and subsequently lyse tumor cells with perforin or by inducing cell apoptosis (26). To reveal the apoptotic effect of ING4 in melanoma A375 cells and $\mathrm{CD}^{+} \mathrm{T}$ cells, dual staining with Annexin V-FITC/7-AAD was used to measure cell apoptosis. It was found that ING4 overexpression could induce melanoma A375 cells and $\mathrm{CD}^{+}$T-cell apoptosis significantly (Fig. 3). Apoptosis may be induced through the extrinsic pathway (activation of cell surface death receptors) or the intrinsic pathway (alterations in the integrity of the mitochondrial membrane that induce the release of cytochrome $c$ ). These pathways converge at the level of the effector caspases (caspase-3, -6, -7 and -8) (27). Once activated, these effector caspases cleave cytoskeletal and nuclear proteins, including PARP, thereby initiating cellular disassembly (27). The present study demonstrated that PARP, caspase- 8 and caspase- 3 were decreased in response to overexpression of ING4 in melanoma cells (Figs. 4 and 5), thereby supporting the premise that cell death occurs in a manner consistent with apoptosis.

Cell death induced by Fas/FasL interaction results in apoptotic signaling in numerous different cell types (28). Fas, FasL and caspase- 8 play important roles in the regulation of apoptosis induction (29). In the present study, the data showed that overexpression of ING4 markedly activated Fas and FasL proteins in melanoma cells and $\mathrm{CD}^{+} \mathrm{T}$ cells (Figs. 4 and 5).

Furthermore, the results suggested that overexpression of ING4 level enhances the apoptosis of A375 cells, which may contribute to a close association with Fas/FasL pathway activation (Figs. 4 and 5). To the best of our knowledge, this study shows that ING4 functions as a tumor suppressor protein in human melanomas. It validates the theory that ING4 can serve as a prognostic marker and a therapeutic target in human melanomas.

In conclusion, in the present study, vectors LV-ING4 and LV-ING4-shRNA were generated and the effect of 
overexpression of ING4 on the apoptosis of human melanoma A375 cells and $\mathrm{CD}^{+} \mathrm{T}$ cells was investigated. The results demonstrated that the high expression level of ING4 could significantly increase melanomas cell apoptosis via the Fas/FasL pathway activation, and also induce the apoptosis of $\mathrm{CD}^{+} \mathrm{T}$ cells. This indicates that ING4 gene therapy could be considered as a novel approach to treat human melanomas.

\section{Acknowledgements}

This study was supported by the National Natural Science Foundation of China (grant no. 81072234).

\section{References}

1. Siegel R, Ma J, Zou Z and Jemal A: Cancer statistics, 2014. CA Cancer J Clin 64: 9-29, 2014.

2. Rubin KM and Lawrence DP: Your patient with melanoma: Staging, prognosis, and treatment. Oncology (Williston Park) 23 (Suppl 8): 13-21, 2009.

3. Grossman D and Altieri DC: Drug resistance in melanoma: Mechanisms, apoptosis, and new potential therapeutic targets. Cancer Metastasis Rev 20: 3-11, 2001.

4. La Porta CA: Mechanism of drug sensitivity and resistance in melanoma. Curr Cancer Drug Targets 9: 391-397, 2009.

5. Alexandrescu DT, Ichim TE, Riordan NH, Marincola FM, Di Nardo A, Kabigting FD and Dasanu CA: Immunotherapy for melanoma: current status and perspectives. J Immunother 33: 570-590, 2010.

6. Korn EL, Liu P-Y, Lee SJ, Chapman JA, Niedzwiecki D, Suman VJ, Moon J, Sondak VK, Atkins MB, Eisenhauer EA, et al: Meta-analysis of phase II cooperative group trials in metastatic stage IV melanoma to determine progression-free and overall survival benchmarks for future phase II trials. J Clin Oncol 26: 527-534, 2008.

7. Gajewski TF: Identifying and overcoming immune resistance mechanisms in the melanoma tumor microenvironment. Clin Cancer Res 12: 2326s-2330s, 2006.

8. Coles AH and Jones SN: The ING gene family in the regulation of cell growth and tumorigenesis. J Cell Physiol 218: 45-57, 2009.

9. Gunduz M, Gunduz E, Rivera RS and Nagatsuka H: The inhibitor of growth (ING) gene family: Potential role in cancer therapy. Curr Cancer Drug Targets 8: 275-284, 2008.

10. Shiseki M, Nagashima M, Pedeux RM, Kitahama-Shiseki M, Miura K, Okamura S, Onogi H, Higashimoto Y, Appella E, Yokota J, et al: p29ING4 and p28ING5 bind to p53 and p300, and enhance p53 activity. Cancer Res 63: 2373-2378, 2003.

11. Li J, Martinka M and Li G: Role of ING4 in human melanoma cell migration, invasion and patient survival. Carcinogenesis 29: 1373-1379, 2008.

12. Friesen C, Herr I, Krammer PH and Debatin K-M: Involvement of the CD95 (APO-1/FAS) receptor/ligand system in drug-induced apoptosis in leukemia cells. Nat Med 2: 574-577, 1996.

13. Hahne M, Rimoldi D, Schröter M, Romero P, Schreier M, French LE, Schneider P, Bornand T, Fontana A, Lienard D, et al: Melanoma cell expression of Fas(Apo-1/CD95) ligand: Implications for tumor immune escape. Science 274: 1363-1366, 1996.
14. Bullani RR, Wehrli P, Viard-Leveugle I, Rimoldi D, Cerottini JC, Saurat JH, Tschopp J and French LE: Frequent downregulation of Fas (CD95) expression and function in melanoma. Melanoma Res 12: 263-270, 2002.

15. Helmbach H, Rossmann E, Kern MA and Schadendorf D: Drug-resistance in human melanoma. Int J Cancer 93: 617-622, 2001.

16. Ekmekcioglu S, Okcu MF, Colome-Grimmer MI, Owen-Schaub L, Buzaid AC and Grimm EA: Differential increase of Fas ligand expression on metastatic and thin or thick primary melanoma cells compared with interleukin-10. Melanoma Res 9: 261-272, 1999.

17. Li X, Cai L, Liang M, Wang Y, Yang J and Zhao Y: ING4 induces cell growth inhibition in human lung adenocarcinoma A549 cells by means of Wnt- $1 / \beta$-catenin signaling pathway. Anat Rec (Hoboken) 291: 593-600, 2008.

18. Clay TM, Custer MC, Sachs J, Hwu P, Rosenberg SA and Nishimura MI: Efficient transfer of a tumor antigen-reactive TCR to human peripheral blood lymphocytes confers anti-tumor reactivity. J Immunol 163: 507-513, 1999.

19. Li X, Cai L, Chen H, Zhang Q, Zhang S, Wang Y, Dong Y, Cheng $\mathrm{H}$ and Qi J: Inhibitor of growth 4 induces growth suppression and apoptosis in glioma U87MG. Pathobiology 76: 181-192, 2009.

20. Wang QS, Li M, Zhang LY, Jin Y, Tong DD, Yu Y, Bai J, Huang Q, Liu FL, Liu A, et al: Downregulation of ING4 is associated with initiation and progression of lung cancer. Histopathology 57: 271-281, 2010.

21. Li X, Zhang Q, Cai L, Wang Y, Wang Q, Huang X, Fu S, Bai J, Liu J, Zhang G, et al: Inhibitor of growth 4 induces apoptosis in human lung adenocarcinoma cell line A549 via Bcl-2 family proteins and mitochondria apoptosis pathway. J Cancer Res Clin Oncol 135: 829-835, 2009.

22. Garkavtsev I, Kozin SV, Chernova O, Xu L, Winkler F, Brown E, Barnett GH and Jain RK: The candidate tumour suppressor protein ING4 regulates brain tumour growth and angiogenesis. Nature 428: 328-332, 2004.

23. Shen JC, Unoki M, Ythier D, Duperray A, Varticovski L, Kumamoto K, Pedeux R and Harris CC: Inhibitor of growth 4 suppresses cell spreading and cell migration by interacting with a novel binding partner, liprin $\alpha 1$. Cancer Res 67: 2552-2558, 2007.

24. Liu Y, Yu L, Wang Y, Zhang Y, Wang Y and Zhang G: Expression of tumor suppressor gene ING4 in ovarian carcinoma is correlated with microvessel density. J Cancer Res Clin Oncol 138: 647-655, 2012.

25. Chan FK-M, Luz NF and Moriwaki K: Programmed necrosis in the cross talk of cell death and inflammation. Annu Rev Immunol 33: 79-106, 2015.

26. Chen G and Emens LA: Chemoimmunotherapy: Reengineering tumor immunity. Cancer Immunol Immunother 62: 203-216, 2013.

27. Fulda S and Debatin KM: Extrinsic versus intrinsic apoptosis pathways in anticancer chemotherapy. Oncogene 25: 4798-4811, 2006.

28. Hohlbaum AM, Moe S and Marshak-Rothstein A: Opposing effects of transmembrane and soluble Fas ligand expression on inflammation and tumor cell survival. J Exp Med 191: 1209-1220, 2000.

29. Nagata S and Golstein P: The Fas death factor. Science 267: 1449-1456, 1995. 